\title{
Combination of a Giant Dissected Ascending Aortic Aneurysm with Multiple Fistulae into the Cardiac Chambers Caused by Prosthetic Aortic Valve Endocarditis
}

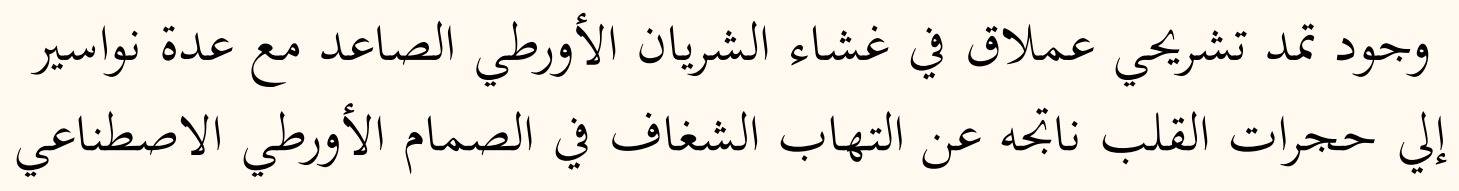

$$
\text { فيريدون سابزي و رضا فرجي }
$$

ABSTR ACT: The combination of a dissected ascending aortic aneurysm (AA) with multiple fistulae to the periaortic root structures is a life-threatening complication that occurs rarely after infective endocarditis of the prosthetic aortic valve. Many risk factors are potentially associated with this complication, including aortic diameter, connective tissue disease of the aortic wall, hypertension and infection. We report a rare case of dissected ascending AA with fistulae to the left atrium and pulmonary artery and a paravalvular leak in a 47-year-old woman with a history of an aortic valve replacement. The patient had presented to the Imam Ali Hospital, Kermanshah, Iran, in January 2015 with clinical features of heart failure. After initially being treated for congestive heart failure, she underwent open-heart surgery via a classic Bentall procedure and double fistula closure. She was discharged 23 days after the operation in good condition. A six-month follow-up showed normal functioning of the composite conduit prosthetic valve and no fistulae recurrence.

Keywords: Aneurysm; Aortic Valve; Fistula; Case Report; Iran.

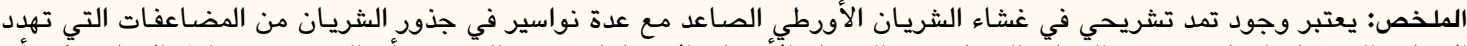

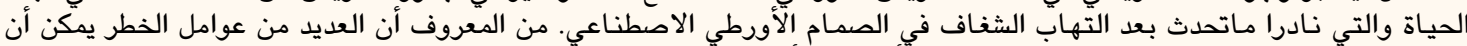

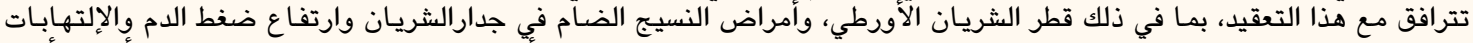

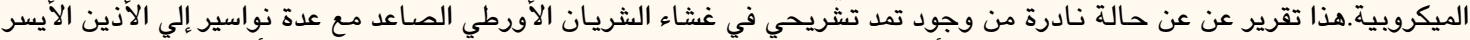

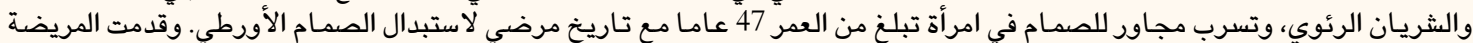

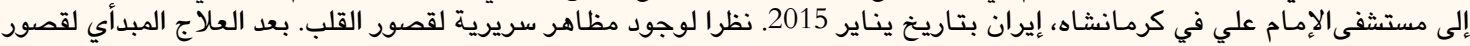

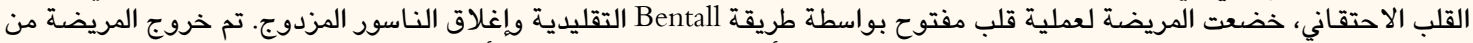

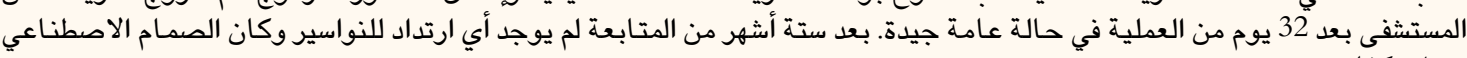

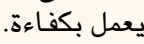

$$
\text { مفتاح الكلمات: تمدد الأوعية الدموية؛ صمام الأورطي؛ ناسور؛ تقرير حالة؛ إيران. }
$$

A NEURYSMAL DILATATION OF A DISSECTED ascending aortic aneurysm (AA) is an uncommon phenomenon in contrast to an isolated dissection or aneurysm. The incidence of this complication is even rarer following aortic valve replacement $(0.2 \%$ and $2 \%){ }^{1}$ The combination of dissected ascending AA with multiple fistulae to the surrounding structures such as the pulmonary artery and left atrium, along with the concomitant presence of paravalvular leaks, is an exceedingly rare complication of prosthetic aortic valve endocarditis. ${ }^{1}$ Research has shown that aortic valve replacement is an independent variable for the development and progression of ascending AAs that may lead to dissection or rupture. ${ }^{2}$
Predisposing factors for the occurrence of postaortic valve replacement aneurysms are well described in the literature. The infected medial aortic wall may be dilated by the haemodynamic burden of aortic regurgitation and a paravalvular leak may be a risk factor for the occurrence of prosthetic aortic valve infective endocarditis (IE). ${ }^{3}$ By inducing a high flow velocity, these paravalvular leaks produce a turbulent flow across the aortic valve which collides with the aortic wall and leads to the gradual dilatation of the ascending aorta. $^{2}$ Related mechanisms can cause ascending AAs in other native valve pathologies such as aortic stenosis or aortic regurgitation, even in the absence of a haemodynamic gradient in patients with 


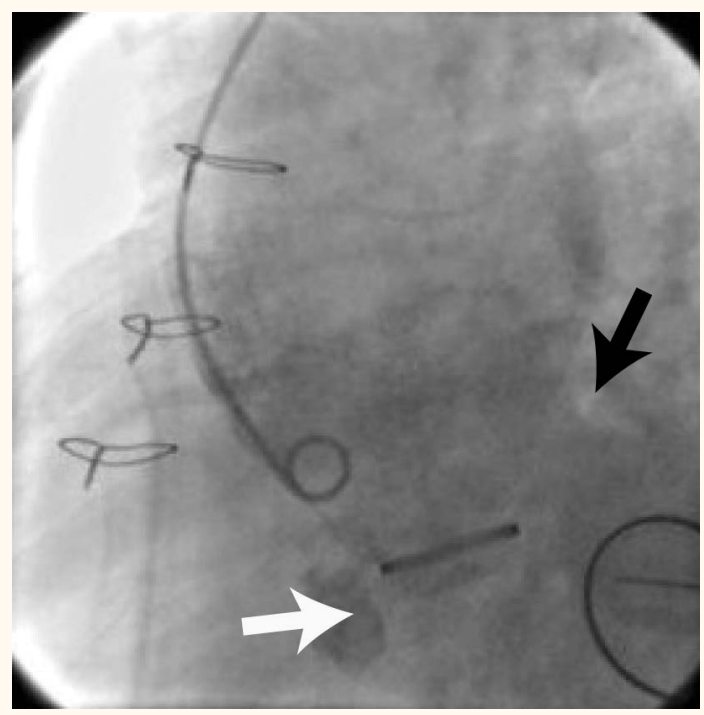

Figure 1: Contrast medium angiograph showing fistulae to the left atrium (white arrow) and pulmonary artery (black arrow) in a 47-year-old woman with a dissected ascending aortic aneurysm.

bicuspid aortic valve. The high volume of regurgitated blood by paravalvular leakage causes turbulent flow in the weakened area of the aortic root and subsequent fistula formation. ${ }^{4}$ This case report describes the successful surgical repair of a giant dissected ascending AA associated with double fistulae to the pulmonary artery and left atrium and the concomitant presence of a small paravalvular leak to the left ventricle.

\section{Case Report}

A 47-year-old woman who had undergone an aortic and mitral valve replacement 10 years previously was admitted to the emergency room of the Imam Ali Hospital, Kermanshah, Iran, in January 2015 with severe dyspnoea and cold perspiration. A physical examination showed pitting oedema on the lower extremities, pulmonary rales and elevated jugular venous pressure. In addition, the patient's skin was cold and damp. The patient history revealed two separate admissions to local hospitals two years earlier for incidences of high fever. She had been treated with appropriate antibiotics during these previous hospital admissions; however, no echocardiographic results were available. She had received oral warfarin and her international normalised ratio was 3-3.5 IU. The patient had had an uneventful postoperative course following her previous primary aortic and mitral valve replacement. At the time of her initial surgery, her native aortic valve was calcified and the diameter of the AA at the sinotubular junction was $4 \mathrm{~cm}$.

At admission, the patient's chest radiography exhibited enlargement of the cardiac silhouette

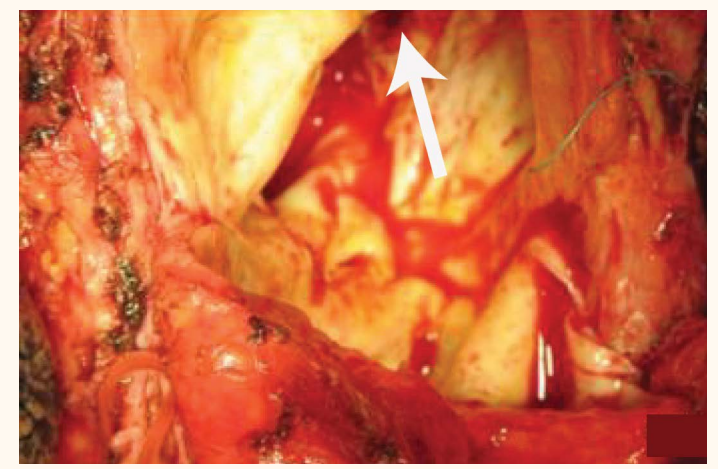

Figure 2: An image showing the fistula to the pulmonary artery (arrow) in a 47-year-old woman with a dissected ascending aortic aneurysm.

and pulmonary congestion. An electrocardiogram showed atrial fibrillation rhythm with rapid ventricular response. A physical examination revealed an irregular heart rhythm, low blood pressure (100/30 mmHg), tachycardia (120 beats per minute), tachypnoea, cyanosis, a prominent jugular vein and a diastolic 3/6 murmur along the left sternal border. The rales were heard diffusely in both lung fields. A transthoracic echocardiogram (TTE) revealed normal functioning of the prosthetic mitral and aortic valve and a giant ascending AA involving the sinuses of Valsalva. The dissection flap was not detected by TTE. The aneurysm's transverse diameter was measured as $7.5 \mathrm{~cm}$ with an aortic root contrast injection [Figure 1].

A transoesophageal echocardiogram (TOE) showed a high- and low-velocity continuous pulse conforming with fistulae from the right and left Valsalva sinuses to the left atrium and pulmonary artery, respectively. Pulmonary artery angiography documented a large left-to-right shunt with a pulmonarysystemic flow ratio of 2:5. Angiography revealed that the coronary arteries were normal. The aortic root angiogram detected the path of the fistulae. The patient was intubated due to severe respiratory dysfunction. She was prepared for urgent cardiac surgery and immediately taken to the operating room.

Due to abnormal preoperative coagulation tests, the patient received fresh frozen plasma. Before the sternotomy, the femoral artery was cannulated; a cardiopulmonary bypass was established after reopening the sternum and right atrial cannulation. A giant AA with severe inflammatory adhesion to the neighbouring organs was observed. The normal diameter of the AA was just below the innominate artery and it was encircled with a tape. After establishing the cardiopulmonary bypass by inducing systemic and local hyperthermia, the giant aorta was transected just above the sinotubular junction. Although a preoperative TTE did not reveal 


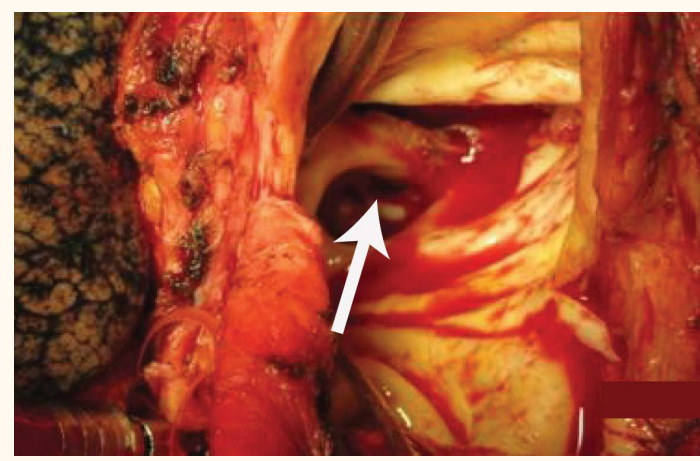

Figure 3: An image showing the left atrial fistula in a 47-year-old woman with a dissected ascending aortic aneurysm. The prosthetic mitral valve was seen deep in the cavity (arrow). The upper curved line shows the native aortic ring after the removal of the prosthetic aortic valve.

a dissection flap, intraoperative inspection revealed a flap in the posterior aortic wall. The prosthetic aortic valve was functionally normal but had a small paravalvular leak to the left ventricle [Figures $2 \& 3$ ]. The orifice of both fistulae tracts in the right and left sinuses of Valsalva and their entrance to the left atrium and pulmonary artery was defined intraoperatively. The fistula tract to the pulmonary artery ended at the main pulmonary artery. The aorto-left atrial fistula was located just over the left atrial roof and opened into the left atrial chamber via the fibrous trigone. Both fistulae were repaired with two interrupted 4-0 pledgeted polypropylene sutures. Intraoperative TOE confirmed that the suture lines of sewing rings on the native fibrous ring were intact. The previous prosthetic aortic valve was removed. A classic Bentall operation was performed using a Carbomedics number 25 valve conduit (Sorin Group, Rome, Italy). Distal anastomosis was performed with a proximal aortic cross-clamp and the patient was weaned from the cardiopulmonary bypass.

The postoperative course was complicated by respiratory and renal failure which was managed accordingly with a tracheostomy, respiratory care, physiotherapy and peritoneal dialysis. On the ninth postoperative day, a tracheotomy was performed and the patient was weaned from the ventilator two days later. She was discharged 23 days after the operation in a good general condition.

\section{Discussion}

Aortic valve replacement is a risk factor for postoperative IE, aortic dilatation, dissection and paravalvular leaks. On the other hand, the presence of a paravalvular leak may be a predisposing factor for IE and subsequent fistulae formation. ${ }^{4}$ The aortic root may become flaccid following the disruption of supporting tissues by primary surgical procedures, such as the releasing of tissue in the aortopulmonary groove, iatrogenic trauma to the aortic wall due to the tip of the needle, extensive decalcification of the aortic ring, calcification of the aortic wall, coronary ostium vault perforation by turbulent flow of the cardioplegia catheter's tip, aortic-mitral fibrous continuity disruption in the primary valve replacement, uncontrolled traction on the aortic wall by the surgeon's assistants or infection of the prosthetic valve and aortotomy suture line. ${ }^{5,6}$

The current patient had three interrelated cardiac characteristics: fistulae, an aneurysm and a history of IE. These complications could be attributed to the patient's previous aortic valve replacement. Complications of the aortic root apparatus following aortic valve replacement is a well-known event and may be associated with high morbidity and mortality. A key complication of aortic valve replacement is ascending aorta dissection, which can lead to various cardiac problems, including ruptures, aneurysms, fistulae formation to a cardiac chamber, haemolytic anaemia, stroke, peripheral emboli and endocarditis. Aorto-cardiac fistulae may be a rare complication of IE.,8

Aortic root IE has been associated with a myriad of complications such as congestive heart failure, stroke, emboli, respiratory failure, pneumonia and dehiscence of a prosthetic valve. ${ }^{5}$ The frequency and type of complications caused by IE have changed with advances in diagnosis and modern antibiotic therapy. Previously common complications of extravalvular cardiac complications of IE-such as fistulae to the cardiac chamber-are infrequent today. ${ }^{8}$ Only $3 \%$ of prosthetic valve IE with Staphylococcus aureus (confirmed by autopsies and retrospective studies) have been associated with cardiac fistulae. It has been postulated that the formation of aorto-cardiac fistulae are caused by a bacterial invasion following a valve replacement; the spread of bacteria from the infected prosthetic valves into the surrounding organs and structures can lead to periannular abscess formation..$^{910}$ The invasion of an abscess into the adjacent tissues can be facilitated by surgical handling or iatrogenic injuries to the aortic wall. ${ }^{6}$ Periprosthetic aortic valve infections with involvement of the aortic ring and Valsalva sinuses may extend upwardly and cause infectious aortitis and a subsequent dilatation or rupture of the aorta, erosion of the fibrous trigone or interventricular septum, which could lead to the formation of aorto-left atrial or left ventricular fistulae. ${ }^{11}$ A periannular abscess in the left Valsalva sinus can sometimes erode into the aortopulmonary groove and lead to an aortopulmonary fistula. ${ }^{7}$ It is 
known that no cardiac chamber can be excluded by IE-induced fistulae and no preponderance from any type of aortic sinus to a specific cardiac chamber can be predicted. ${ }^{3,4,8-11}$

\section{Conclusion}

The combination of aorto-cardiac fistulae with a dissected ascending AA is a very rare and fatal complication of aortic valve replacement. Both complications may be caused by IE or they may each have a specific aetiology. The diagnosis of aorto-cardiac fistulae with a dissected ascending AA should be considered in patients with a history of aortic valve replacement and in those admitted with a diastolic murmur, congestive heart failure or shock in the setting of a post-aortic valve replacement AA. Prompt surgical intervention is necessary for patient survival.

\section{References}

1. Holmes DR Jr, Brennan JM, Rumsfeld JS, Dai D, O'Brien SM, Vemulapalli $\mathrm{S}$, et al. Clinical outcomes at 1 year following transcatheter aortic valvereplacement.JAMA2015;313:1019-28. doi: 10.1001/jama.2015.1474.

2. Sreedharan M, Baruah B, Dash PK. Aorta-right atrial tunnel: A novel therapeutic option. Int J Cardiol 2006; 107:410-12. doi: 10.1016/j.ijcard.2005.01.056.

3. Girdauskas E, Disha K, Borger MA, Kuntze T. Long-term prognosis of ascending aortic aneurysm after aortic valve replacement for bicuspid versus tricuspid aortic valve stenosis. J Thorac Cardiovasc Surg 2014; 147:276-82. doi: 10.1016/j.jtcvs .2012.11.004.
4. Tsutsumi K, Inoue Y, Hashizume K, Kimura N, Takahashi R. Risk factor analysis for acute type A aortic dissection after aortic valve replacement. Gen Thorac Cardiovasc Surg 2010; 58:601-5. doi: 10.1007/s11748-010-0658-z.

5. Vahanian A, Baumgartner H, Bax J, Butchart E, Dion R, Filippatos G, et al. Guidelines on the management of valvular heart disease: The Task Force on the Management of Valvular Heart Disease of the European Society of Cardiology. Eur Heart J 2007; 28:230-68.

6. Yilmaz A, Dessing T, Botta L. Ascending aorta impending rupture: Successful surgical management in a patient with type IV Ehlers-Danlos syndrome. J Cardiovasc Med (Hagerstown) 2010; 11:610-12. doi: 10.2459/JCM.0b013e3283314176.

7. Roseborough GS, Williams GM. Marfan and other connective tissue disorders: Conservative and surgical considerations. Semin Vasc Surg 2000; 13:272-82.

8. Benedik J, Pilarzcyk K, Wendt D, Price V, Tsagakis K, Perrey M, et al. Is there any difference in aortic wall quality between patients with aortic stenosis and those with regurgitation? Eur J Cardiothorac Surg 2013; 44:754-9. doi: 10.1093/ejcts/ezt123.

9. Tzemos N, Therrien J, Yip J, Thanassoulis G, Tremblay S, Jamorski MT, et al. Outcomes in adults with bicuspid aortic valves. JAMA 2008; 300:1317-25. doi: 10.1001/jama.300.11. 1317.

10. McKellar SH, Michelena HI, Li Z, Schaff HV, Sundt TM 3rd. Long-term risk of aortic events following aortic valve replacement in patients with bicuspid aortic valves. Am J Cardiol 2010; 106:1626-33. doi: 10.1016/j.amjcard.2010.07.043.

11. Matsuhisa H, Obo H, Nakagiri K, Mokohara N, Shida T. Aortoright atrial fistula caused by type A aortic dissection. Ann Thorac Surg 2004; 78:2173-5. doi: 10.1016/j.athoracsur.2003. 08.007. 